\title{
QTL Analysis for Downy Mildew Resistance in Cucumber Inbred Line PI 197088
}

Lixia Li, Huiqiang He, and Zhirong Zou, ${ }^{\dagger}$ College of Horticulture, Northwest A\&F University, Yangling, China; Key Laboratory of Protected Horticultural Engineering in Northwest, Ministry of Agriculture, Yangling, China; and State Key Laboratory of Crop Stress Biology for Arid Areas, Yangling, China; and Yuhong Li, ${ }^{\dagger}$ College of Horticulture, Northwest A\&F University, Yangling, China

\begin{abstract}
Downy mildew (DM), caused by Pseudoperonospora cubensis, is one of the major foliar diseases prevailing in cucumber-growing areas. The mechanism of DM resistance in cucumber, particularly the plant introduction (PI) 197088 from India, is presently unclear. Quantitative trait locus (QTL) mapping is an efficient approach to studying DM resistance genes in cucumber. In this study, we performed QTL mapping for DM resistance in PI 197088 with $183 \mathrm{~F}_{2}$-derived $\mathrm{F}_{3}\left(\mathrm{~F}_{2: 3}\right)$ families from the cross between PI 197088 (DM resistant) and Changchunmici (DM susceptible). A linkage map was constructed using 141 simple sequence

repeat markers. Phenotypic data were collected from seven independent experiments. In total, five QTL were detected on chromosomes 1, 3, 4, and 5 with DM resistance contributed by PI 197088. The QTL on chromosome $4, d m 4.1$, was reproducibly detected in all indoor experiments, which could explain $27 \%$ of the phenotypic variance detected. Additionally, $d m 1.1$ and $d m 5.2$ showed moderate effects, while $d m 3.1$ and $d m 5.1$ were minor-effect QTL. This study revealed the unique genetic architecture of DM resistance in PI 197088, which may provide important guidance for efficient use in cucumber breeding for DM resistance.
\end{abstract}

Cucumber (Cucumis sativus L.) is an important vegetable crop widely grown around the world, yet it is susceptible to many diseases and pests during growth. Downy mildew (DM), caused by the obligate biotrophic oomycete Pseudoperonospora cubensis (Berk. \& M. A. Curtis) Rostoyzev, is one of the major foliar diseases in cucumber. $P$. cubensis can spread by wind, rain, irrigation water, and seed (Call et al. 2012a; Cohen et al. 2014). In the early stage of DM development, watery lesions develop on the abaxial leaf surface and, as the condition progresses, chlorotic lesions appear on the adaxial leaf surface. In the later stages of DM development, the upper side of the leaf turns necrotic, while a thick layer of black mold is formed on the underside; eventually, the leaf withers and loses function (Savory et al. 2011). Available methods for DM prevention and control are environmental control, fungicide application, and biotechnological approaches. However, the regulation and control of the environment is time consuming and laborious, with limited effectiveness (Ojiambo et al. 2015). Long-term use of fungicides may cause fungicide resistance and damage to the ecological environment (Cohen et al. 2015; Wallace et al. 2015). Studying the inheritance pattern of DM resistance and breeding DM-resistant varieties of cucumber are currently the most cost-effective way of DM prevention.

Screening for DM resistance in cucumber germplasm has been ongoing for decades. In the United States, screening for DM-resistant lines was started as early as 1942 (Jenkins 1942). Barnes and Epps (1954) identified a resistant plant introduction (PI) line PI 197087 carrying a recessive resistance gene, $d m-1$. Subsequently, numerous DM-resistant varieties carrying $d m-1$ were cultivated, such as Gy4, Chipper, and the Marketmore series (Call et al. 2012a; Wehner and Shetty 1997). Over the following five decades, cucumber DM was

${ }^{\dagger}$ Corresponding authors: Z. Zou; E-mail: zouzhirong2005@hotmail.com; and Y. Li; E-mail: liyuhong73@nwsuaf.edu.cn

L. Li and H. He contributed equally to this study.

Funding: This study was supported by the Key Technology Integration and Demonstration of Horticultural Crops Growing on Non-Cultivated Land in Northwest China (number K312021301).

*The $\boldsymbol{e}$-Xtra logo stands for "electronic extra" and indicates that three supplementary figures and three supplementary tables are published online.

Accepted for publication 14 December 2017.

() 2018 The American Phytopathological Society well controlled. However, new physiological races of $P$. cubensis emerged in 2004, which overcame the resistance conferred by $d m-1$ and caused widespread damage to cucumber production in the United States (Holmes et al. 2015). Based on multiyear and multilocation screening tests among over 1,300 cucumber collections, Call et al. (2012b) identified PI 197088, PI 330628, and PI 605996 that were highly resistant to DM. Some DM-susceptible plants were observed in the $\mathrm{F}_{2}$ from crosses among the three PI lines, suggesting possibly different genetic bases of DM resistances in these germplasms (VandenLangenberg 2015). Thus, identification of DM resistance quantitative trait loci (QTL) in these materials may help encourage better use of them in cucumber breeding.

Several studies on QTL mapping for DM resistance in cucumber have been conducted. Using an $\mathrm{F}_{2: 3}$ population, Zhang et al. (2013) detected five QTL on three cucumber chromosomes that contributed DM resistance ( $d m 1.1, d m 5.1, d m 5.2, d m 5.3$, and $d m 6.1)$ in the north China type cucumber line K8. Pang et al. (2013) evaluated an $\mathrm{F}_{2}$ population derived from IL52 (DM resistant) $\times$ Changchunmici (DM susceptible) and identified six QTL for DM resistance. PI 197085 is among the best resistant lines against $P$. cubensis (Call et al. 2012b). Szczechura et al. (2015) suggested that DM resistance in this line is quantitative and QTL for DM resistance were located on cucumber chromosome 5 at $24^{\circ} \mathrm{C}$.

Recently, Wang et al. (2016) collected phenotypic data of the $\mathrm{F}_{2: 3}$ families from a cross between two inbred lines (DM-resistant WI7120 $\times$ DM-susceptible 9930) across four different environments in three countries for 2 years. They identified four stable QTL for DM resistance on cucumber chromosomes 2, 4, 5, and 6, respectively, with DM resistance always inherited from WI7120. In South Korea, Win et al. (2016) constructed QTL mapping in an $\mathrm{F}_{2}$ population with TH118FLM as the resistant parent and detected five QTL (dm2.2, $d m 4.1, d m 5.1, d m 5.2$, and $d m 6.1)$ using a next-generation highthroughput sequencing. Overall, many QTL associated with DM resistance have been identified in cucumber across seven chromosomes. However, the quantity and distribution of QTL for DM resistance were different in the above studies, with various resistance resources, evaluation traits, and environmental conditions. Thus, no consensus has been reached on the complex mechanism of DM resistance in cucumber.

PI 197088 is known as a cucumber line with high resistance to powdery mildew (PM) and DM. QTL for PM resistance in this line have been reported (Fukino et al. 2013; Sakata et al. 2006). Regarding the mechanism of DM resistance in PI 197088, Caldwell et al. (2014) constructed a population with PI 197088 as the resistant parent and mapped three QTL on chromosomes 2, 4, and 5, respectively, 
with DM resistance from PI 197088. CS-PMR1 is a cucumber line with high resistance to DM derived from PI 197088. Yoshioka et al. (2014) performed a QTL analysis for DM resistance in a recombinant inbred line $(\mathrm{RIL})$ population from the cross CS-PMR $1 \times$ Santou (with intermediate DM resistance). Ten QTL were mapped on chromosomes $1,3,5,6$, and 7, seven of which were contributed by CS-PMR1. Furthermore, VandenLangenberg (2015) constructed a RIL population from the cross PI $197088 \times$ Coolgreen and detected three candidate QTL for DM resistance on chromosomes 4 and 5. The two studies suggested that DM resistance in PI 197088 is under the control of a complex genetic system. Because the progression of DM symptoms is highly influenced by the environment, multilocation and multiyear phenotyping is recommended. Compared with field observations, indoor experiments can be used to reduce the environmental variance.

To better understand the genetic basis of DM resistance in PI 197088 cucumber, we constructed an $\mathrm{F}_{2: 3}$ population for the cross between PI 197088 and the susceptible line Changchunmici. QTL analysis was performed with phenotypic data collected from multiple environments over 2 years and the results are reported below.

\section{Materials and Methods}

Plant materials. The $\mathrm{F}_{1}$ generation was derived from a cross between two cucumber inbred lines, PI 197088 from India (with a high level of stable resistance to DM) (Call et al. 2012b) and Changchunmici from China (susceptible to DM). An $\mathrm{F}_{2}$ population was obtained by selfing the $F_{1}$ progeny, and $F_{2}$ plants were self-pollinated to produce $\mathrm{F}_{2}$-derived $\mathrm{F}_{3}\left(\mathrm{~F}_{2: 3}\right)$ families. In total, $183 \mathrm{~F}_{2}$ individuals was used in genetic map construction, and the corresponding $\mathrm{F}_{2: 3}$ families were used in phenotypic evaluation and QTL analysis for DM resistance in cucumber.

Inoculation experiments. $P$. cubensis used in the experiments was collected from naturally infected cucumber leaves displaying only DM symptoms (Holmes et al. 2015). The diseased leaves were washed with sterile water to prepare spore suspension. The identity of $P$. cubensis was determined by morphological observation under a microscope (Colucci and Holmes 2010). The spore suspension was sprayed at a rate of $1 \times 10^{5}$ sporangia/ml with a handheld sprayer.

Three indoor experiments, denoted as Exp1, Exp2, and Exp3, were performed in May 2013, June 2014, and November 2014, respectively. Cucumber seedlings were grown in sterile plastic trays ( 7.5 by 7.5 by $8 \mathrm{~cm}$ ) with one plant per tray and plant spacing of $5 \mathrm{~cm}$. Each tray was filled with a mixture of peat, perlite, and vermiculite $(3: 1: 1, \mathrm{vol} / \mathrm{vol} / \mathrm{vol})$. The seedlings were watered daily and fertilized with the Hoagland nutrition solution (Hoagland and Arnon 1950) once every 3 days. Seedlings with fully expanded first true leaves were inoculated in a climate-controlled room of Northwest A\&F University (Yangling, Shaanxi, China) with cycles of $12 \mathrm{~h}$ of light and $12 \mathrm{~h}$ of darkness, and the light intensity was set at $200 \mu \mathrm{mol} \mathrm{m}{ }^{-2} \mathrm{~s}^{-1}$. The temperature was set at constant at $22^{\circ} \mathrm{C}$. All three experiments had three replications with a randomized complete block design. Each replication included two parental lines and their $F_{1}$ and $F_{2: 3}$ families that had $32,32,32$, and 5 individuals, respectively. Only the first true leaves were spray inoculated to ensure a uniform distribution of spore suspension on both sides of the leaf. The inoculated plants were kept in the dark for $24 \mathrm{~h}$ at $22^{\circ} \mathrm{C}$ under $100 \%$ relative humidity, and then maintained at conditions described above. Phenotypic data were collected on the first true leaves of inoculated plants 7 to 10 days after inoculation (dai).

Greenhouse inoculation assays, denoted as Exp4 and Exp5, were conducted in May and September 2013, respectively. Open field experiments, denoted as Exp6 and Exp7, were conducted in September 2013 and October 2014, respectively. In these four experiments, phenotypic data of inoculation responses were collected from $P$. cubensis for parental lines and $183 \mathrm{~F}_{2: 3}$ families. All four experiments used a randomized complete block design with two replications and five plants per replication. Cucumber seedlings were grown in sterile plastic trays $(10$ by 10 by $8.8 \mathrm{~cm}$ ), with one plant per tray and plant spacing of $15 \mathrm{~cm}$. Each tray was filled with a mixture of peat, perlite, and vermiculite $(3: 1: 1, \mathrm{vol} / \mathrm{vol} / \mathrm{vol})$. Seedlings were placed in a plastic greenhouse or experimental plots at the Northwest A\&F University experimental farm. The seedlings were fertilized with only half the normal concentration of the Hoagland nutrition solution (Hoagland and Arnon 1950) daily. At dusk, plants were inoculated at the four-true-leaf stage by misting both sides of leaves with the sporangial suspension until runoff using a handheld sprayer. For the first two nights of inoculation, disease development was encouraged by using a humidifier (ZS-50Z, Hangzhou, China) between 8 P.M and 6 A.M. in greenhouse, and surface irrigation by drip irrigation pipes (Yirun, Hebei, China) in the field, with the drippers $15 \mathrm{~cm}$ apart for $3 \mathrm{~h}$. The seedlings were scored at 15 dai. The average temperature was $20,20,18$, and $14^{\circ} \mathrm{C}$ in Exp4 (range from 14 to $34^{\circ} \mathrm{C}$ ), Exp5 (range from 12 to $32^{\circ} \mathrm{C}$ ), Exp6 (range from 10 to $32^{\circ} \mathrm{C}$ ), and Exp7 (range from 6 to $25^{\circ} \mathrm{C}$ ), respectively.

Phenotypic data analysis. We performed phenotypic evaluation on the inoculated plants based on DM development on the first true leaves in the indoor experiments, as well as the second to fourth leaves in the greenhouse and field experiments. Based on the percentage of symptomatic leaf area, a scale of 1 to 9 disease scoring system was used, where $1=0$ to $10,2=11$ to $20,3=21$ to $30,4=31$ to 40 , $5=41$ to $50,6=51$ to $60,7=61$ to $70,8=71$ to 80 , and $9=81$ to $100 \%$ area symptomatic or withered. Thus, 1 was the most resistant and 9 the most susceptible (Supplementary Fig. S1). A disease index (DI) was then calculated (Zhang et al. 2013) for statistical analysis. The DI mean from each $\mathrm{F}_{3}$ family was used for QTL analysis. Data analysis was performed in $\mathrm{R}$ (version 3.1.1; http://www.r-project. org/) but a calculation (means of disease scores) was performed in Excel 2016 (Microsoft Corp., Redmond, WA).

Simple sequence repeat marker analysis. A genetic map was developed using cucumber simple sequence repeat (SSR) markers according to Cavagnaro et al. (2010) and Yang et al. (2013). Polymorphism screening of these SSR markers was first conducted between PI 197088 and Changchunmici; polymorphic markers were then applied to the segregating population for map construction and QTL analysis.

DNA extraction, polymerase chain reaction (PCR) amplification of SSR markers, and gel electrophoresis were performed according to the method of $\mathrm{Li}$ et al. (2013). Linkage analysis was conducted using JoinMap 4.0 (Van Ooijen 2006). Genetic map construction was performed using regression mapping and the Kosambi mapping function (Kosambi 1943). Linkage groups were assigned at logarithm of odds (LOD) $\geq 4$.0. Common markers shared with the high-density maps published by Ren et al. (2009) and Yang et al. (2013) were used to declare the relationship between linkage groups and cucumber chromosomes.

QTL analysis. QTL analysis was performed using the composite interval mapping (CIM) model in WinQTL Cart 2.5 (Wang et al. 2012). The following parameters were used: model $=6$, regression method $=$ forward and backward, windows size $=1$ centimorgan $(\mathrm{cM})$, and control marker number $=5$. The phenotypic variance $\left(R^{2}\right)$ was calculated for confirmed significant QTL. The confidence interval of QTL was determined using the 2-LOD support interval method.

QTL analysis was also performed using the R/qtl program with the multiple-QTL model (MQM) and CIM model (Arends et al. 2010; Broman and Sen 2009; Broman et al. 2003). The parameter settings followed Wang et al. (2016) and Yoshioka et al. (2014). The significant LOD threshold for QTL was calculated by 1,000 permutations at the significance threshold of 0.05 .

QTL that explained more than $15 \%$ phenotypic variance were considered as major-effect QTL (He et al. 2013). Based on the linkage map, the locations of QTL for DM resistance on each chromosome was plotted using MapChart 2.3 (Voorrips 2002).

\section{Results}

Phenotypes of DM resistance reaction. The DM symptom development in test materials was examined 7 to 10 dai. The means of DI for parental lines, $\mathrm{F}_{1}$, and $\mathrm{F}_{2: 3}$ families are presented in Table 1. PI 197088 was highly resistant to DM in all experiments, despite some variations in DI means across environments (from $5.14 \pm 0.38$ to 
$20.97 \pm 8.05$ ), whereas Changchunmici was consistently susceptible in all conditions (DI mean $>80$ ). DI mean of the $\mathrm{F}_{2: 3}$ families was, in general, intermediate between the parental lines in each experiment. Effects of environments on DM disease development were obvious. Disease severity of plants differed in the seven experiments, with the DI mean in Exp1 the highest and that of Exp6 the lowest. The frequency distribution of DI means among $183 \mathrm{~F}_{3}$ families was largely normal (Fig. 1), suggesting that DM resistance in PI 197088 was controlled by polygenes. The mean DI of the two parental lines was close to the respective ends but transgressive segregation was found in individual lines of the $\mathrm{F}_{2: 3}$ families. Correlations among DI means were, in general, positive and significant $(P<0.01)$. The correlations were higher in the same conditions and weaker in different environments (Supplementary Fig. S2).

Genetic map construction. Among 420 cucumber SSR markers screened, 141 (33.57\%) were polymorphic between PI 197088 and Changchunmici, which were applied to $183 \mathrm{~F}_{2}$ plants for genotyping. Detailed information of all markers placed on this map is presented in Supplementary Table S1. The resulting genetic map had 141 marker loci with a total map length of $635.25 \mathrm{cM}$ and an average interval of $4.51 \mathrm{cM}$ (Table. S2). Compared with the genetic maps of Ren et al. (2009) and Yang et al. (2013), there were 53 and 51 common markers in our genetic map, respectively. These common markers were used to assign linkage groups to seven chromosomes of cucumber. On the genetic map (Supplementary Table S2; Fig. 2), chromosome 3 was the longest $(116.97 \mathrm{cM})$ and chromosome 7 was the shortest $(54.41 \mathrm{cM}$, 10 marker loci).

QTL analysis for DM resistance. Phenotypic data of $183 \mathrm{~F}_{3}$ families collected from all seven environments (Exp1 to Exp7) were used in QTL analysis of DM resistance in PI 197088. No significant differences were found when different QTL analysis methods were used. However, the QTL detected under different experimental conditions were variable. Details of each QTL, including detection method, chromosome, position, LOD, $R^{2}$, additive effect, dominant effect, and marker interval, are provided in Supplementary Table S3 and Supplementary Fig. S3.

Five different QTL were detected in the seven experiments, which were located on chromosomes 1, 3, 4, and 5; their LOD ranged from 3.02 to 14.41 . These QTL cumulatively explained 7 to $27 \%$ of observed phenotypic variance (Table 2). The major-effect QTL, dm4.1 on chromosome 4, was detected in four experiments with high LOD support (14.41) that could explain $27 \%$ phenotypic variance. The flanking markers of $d m 4.1$ were UW084520 and UW042029. In all indoor experiments, despite the differences in peak position, LOD support, and $R^{2}$ values, the physical intervals of $d m 4.1$ overlapped, suggesting $d m 4.1$ was a highly stable, major-effect QTL for DM resistance in PI 197088.

Two QTL- $d m 1.1$, with $R^{2}$ of $10.62 \%$, and $d m 5.2$, with $R^{2}$ of $12.46 \%$ - were considered moderate-effect QTL. The $d m 1.1$ was detected in Exp4 to Exp7, and the flanking markers were UW084399 and UW083946. The $d m 5.2$ was detected in Exp3 and Exp6 with CIM. The $d m 5.1$ with $R^{2}$ of $7.6 \%$ was detected only once, which was a minor-effect QTL; this QTL was located between UW084534 and

Table 1. Means and standard deviations of downy mildew disease index of two parental lines (PI $197088 \times$ Changchunmici) and their $F_{1}$ and $F_{2: 3}$ families from seven experiments

\begin{tabular}{lrccc}
\hline Experiment $^{\mathbf{a}}$ & PI 197088 & Changchunmici & $\mathbf{F}_{\mathbf{1}} \mathbf{b}$ & $\mathbf{F}_{2: 3} \mathbf{c}$ \\
\hline Exp1 & $20.44 \pm 7.93$ & $90.56 \pm 7.48$ & $71.94 \pm 8.60$ & $60.41 \pm 15.99$ \\
Exp2 & $15.83 \pm 6.03$ & $80.28 \pm 9.12$ & $65.00 \pm 8.80$ & $46.39 \pm 23.02$ \\
Exp3 & $17.44 \pm 8.15$ & $85.83 \pm 8.60$ & $67.50 \pm 9.09$ & $48.48 \pm 18.06$ \\
Exp4 & $11.62 \pm 4.16$ & $81.22 \pm 8.64$ & - & $47.00 \pm 17.34$ \\
Exp5 & $18.11 \pm 5.37$ & $87.41 \pm 5.89$ & - & $50.06 \pm 11.88$ \\
Exp6 & $5.14 \pm 0.38$ & $80.13 \pm 8.99$ & - & $35.65 \pm 19.12$ \\
Exp7 & $20.97 \pm 8.05$ & $83.76 \pm 9.03$ & - & $52.72 \pm 24.51$ \\
\hline
\end{tabular}

${ }^{a}$ Exp1, Exp2, and Exp3 were performed indoors; Exp4 and Exp5 were performed in a greenhouse; and Exp6 and Exp7 were performed in the field.

${ }^{\mathrm{b}}$ Symbol - indicates data not available.

${ }^{\mathrm{c}}$ Grand family means of disease index.
UW085065. Similarly, the $d m 3.1$ with $R^{2}$ of $7.00 \%$ was only detected in Exp3 using R/qtl (Table 2).

\section{Discussion}

QTL for DM resistance in cucumber are affected by many factors. Environmental conditions have a strong influence on the speed and
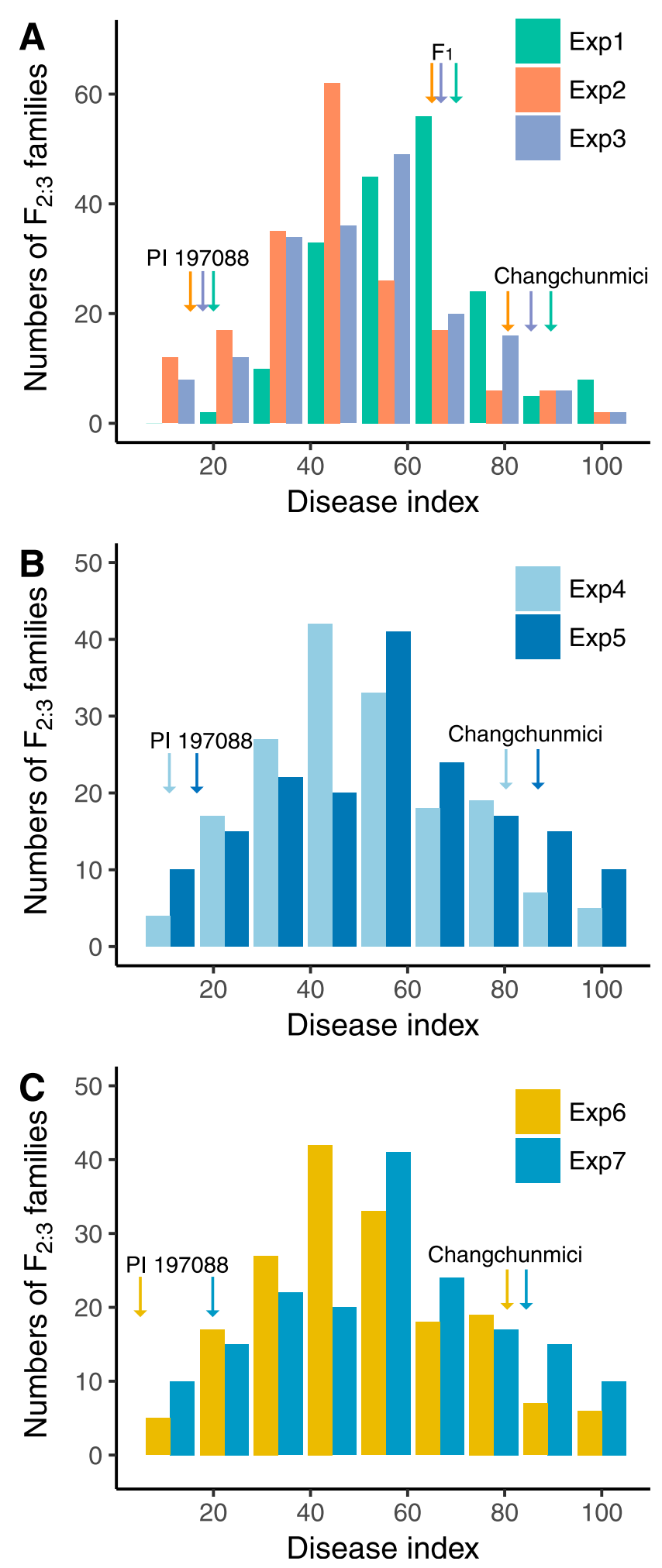

Fig. 1. Frequency distribution of disease index means to cucumber downy mildew in $\mathrm{F}_{2: 3}$ families (PI $197088 \times$ Changchunmici) in A, indoor experiments (Exp1 to Exp3); B, greenhouse experiments (Exp4 and Exp5); and C, field experiments (Exp6 and Exp7). Each has a continuous distribution ranging from resistant (left) to susceptible (right). Arrows indicate the corresponding values of PI 197088, Changchunmici, and their $F_{1}$ from different experiments. 
severity of DM symptom development. Previous studies on DM in cucumber were conducted mainly in plastic greenhouses or open fields (Pang et al. 2013; VandenLangenberg 2015; Wang et al. 2016; Win et al. 2016; Yoshioka et al. 2014), making it difficult to precisely control the environmental conditions and disease uniformity.

In the present study, three experiments (Exp1 to Exp3) were first conducted in a climate-controlled room with better control of temperature, humidity, and light conditions. Artificial inoculation of $P$. cubensis was used to ensure uniform DM development. These measures assured collection of accurate phenotypic data. Based on the comparison of QTL results in different experiments, we found that $d m 4.1$ could be reproducibly detected in the indoor conditions (Exp1 to Exp3) but not in Exp4, Exp6, or Exp7. Another QTL, $d m 1.1$, showed the opposite trend. It is possible that the greenhouse and field environments were similar. These findings provided evidence that the environmental conditions had a strong effect on the severity of DM infection.

We observed that the QTL results were affected by different detection methods. Nearly identical results were obtained using three methods in Exp1. In Exp6, for example, $d m 1.1$ and $d m 5.1$ were detected by R/qtl-CIM, another two QTL were detected by WinQTL Cart 2.5-CIM, and no QTL was detected by R/qtl-MQM. The varying ability to detect QTL by different methods has also been reported in previous studies in cucumber (Weng et al. 2015; Yoshioka et al. 2014). Thus, using multiple methods for detecting QTL is a better way for future experiments to study DM resistance in cucumber.

Compared with a single-spore culture, the $P$. cubensis population derived from a naturally infected cucumber field is more pathogenic (Cohen et al. 2015; Pais et al. 2013; Savory et al. 2011). In this study, we collected $P$. cubensis from naturally diseased cucumber leaves in the greenhouse. The identity of $P$. cubensis was determined by morphological observation. The vitality and pathogenicity of the pathogen might vary with different statuses and phases of disease development. Nonetheless, $d m 1.1$ and $d m 4.1$ were detected repeatedly, indicating minor differences of the pathogen in this study.

A number of studies have indicated race differentiation of the P. cubensis pathogen worldwide (Angelov et al. 2000; Lebeda and Cohen 2011; Lebeda et al. 2002; Naegele et al. 2016; Savory et al. 2011; Shetty et al. 2002), which may affect DM resistance screening in cucumber. In several QTL mapping studies for PI 190788, the QTL detected were somewhat varied (VandenLangenberg 2015; Yoshioka et al. 2014) (present study). We detected DM resistance QTL on cucumber chromosomes 1,3, and 5, which was largely consistent with results by Yoshioka et al. (2014) in Japan. Very likely, the DM pathogen profiles or virulence between Japan and China are more similar compared with those in the United States. However, additional evidence is needed to confirm this.

There were some differences between our study and Yoshioka et al. (2014). For instance, we used resistant and susceptible materials as the parents, and the resistance in the QTL detected was derived from the resistant parent (PI 197088). However, Yoshioka et al. (2014) used highly resistant (CS-PMR1) and moderately resistant (Santou) materials as the parents, and the resistance in the QTL was derived from both the parents. Additionally, although both studies used the first true leaves in the indoor experiments, the environmental temperature settings were different $\left(25\right.$ and $\left.22^{\circ} \mathrm{C}\right)$. All these could affect the results of QTL detection for DM resistance in cucumber.

Moreover, the development of DM seems to be related to growth stage of plants (Palti and Cohen 1980), which affects the detection of QTL for DM resistance (VandenLangenberg 2015; Yoshioka et al. 2014). The same associations were observed in the present study. Taken together, we believe that cucumber DM resistance depends

Table 2. Quantitative trait loci (QTL) analysis for downy mildew resistance in cucumber based on $\mathrm{F}_{2: 3}$ populations (PI $197088 \times$ Changchunmici)

\begin{tabular}{|c|c|c|c|c|c|c|c|}
\hline \multirow{2}{*}{$\frac{\text { QTL }}{d m 1.1}$} & \multirow{2}{*}{$\begin{array}{c}\text { Chromosome } \\
1\end{array}$} & \multirow{2}{*}{$\frac{\text { Position (cM) }}{67.01-90.00}$} & \multicolumn{2}{|c|}{ Marker interval } & \multirow{2}{*}{$\frac{\text { Logarithm of odds }}{3.02-6.15}$} & \multirow{2}{*}{$\frac{\boldsymbol{R}^{2}(\%)^{\mathbf{a}}}{10.62}$} & \multirow{2}{*}{$\frac{\text { Experiment }}{4,5,6,7}$} \\
\hline & & & UW084399 & UW083946 & & & \\
\hline$d m 3.1$ & 3 & $84.00-84.70$ & SSR04570 & SSR15029 & $3.03-4.14$ & 7.00 & 2 \\
\hline$d m 4.1$ & 4 & $30.21-44.00$ & UW084520 & UW042029 & $4.00-14.41$ & 27.00 & $1,2,3,5$ \\
\hline$d m 5.1$ & 5 & 19.31 & UW084534 & UW085065 & 3.32 & 7.60 & 6 \\
\hline$d m 5.2$ & 5 & $82.81-97.60$ & UW084347 & UW063445 & $3.73-4.20$ & 12.46 & 3,6 \\
\hline
\end{tabular}

a Selection of maximum value from different models in Exp1 to Exp7 as percentage of phenotypic variance explained $\left(R^{2}\right)$.

Chr1

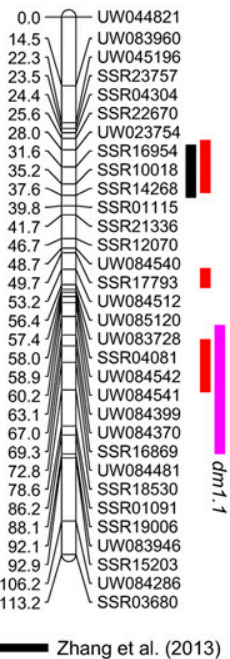

Chr2

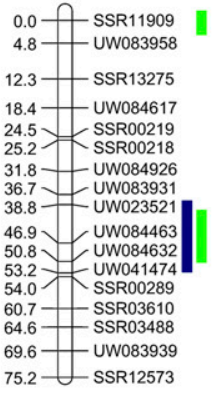

Chr3

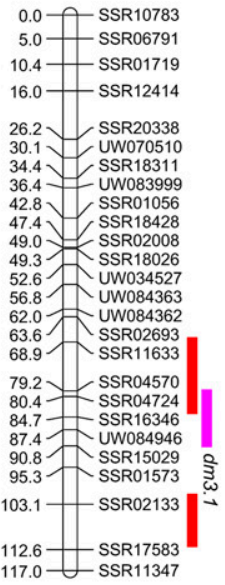

Chr4

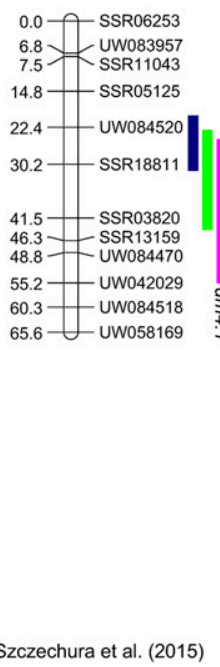

Chr5

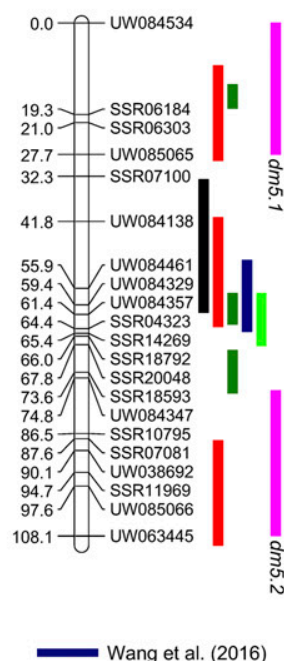

Chr6

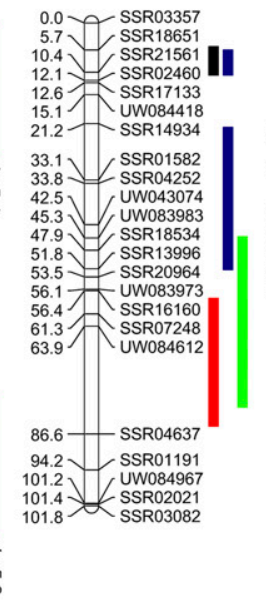

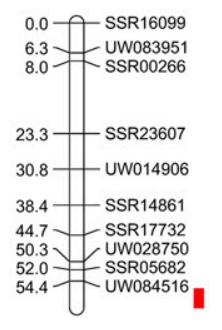

Fig. 2. Genetic map of $F_{2}$ populations derived from cucumber PI $197088 \times$ Changchunmici and locations of quantitative trait loci (QTL) for downy mildew resistance. This map only contains several QTL detected from 2013 to 2016, which were published in previous studies and this study. 
not only on the genetic resistance of the host but also on other factors (e.g., detection method, environmental condition, pathogen race, and plant growth stage) that affect the detection of QTL.

Genetic basis of DM resistance in PI 197088. Combined with previous QTL mapping studies, we found that QTL associated with DM resistance could be detected on seven chromosomes of cucumber (Fig. 2); however, there were inconsistencies between different studies. The $d m 1.1$ detected in the present study likely corresponded to the resistance QTL $d m 1.3$ on chromosomes 1 in Yoshioka et al. (2014). QTL $d m 3.1$ detected herein overlapped with $d m 3.2$ detected in Yoshioka et al. (2014) using the same resistant source (PI 197088). Neither $d m 1.1$ nor $d m 3.1$ has been reported in studies of other cucumber materials, indicating that chromosomes 1 and 3 may include QTL regions for DM resistance specifically related to PI 197088.

We detected a major-effect QTL on chromosome 4, which overlapped in a wide range with $d m 4.1$ detected by Win et al. (2016) using the DM-resistant line TH118FLM and was close to the location of $d m 4.1$ detected by Wang et al. (2016) from WI7120 (PI 330628). We speculate that at least one cucumber DM resistance gene may exist in this region. Although $d m 4.1$ was reproducibly detected in multiple environments in our study, it was not detected by Yoshioka et al. (2014). Temperature might be a factor for this discrepancy; we used a constant temperature environment $\left(22^{\circ} \mathrm{C}\right)$ in the present study.

Chromosome 5 seems to harbor multiple QTL for DM resistance in cucumber (Fig. 2). In our study, $d m 5.1$ and $d m 5.2$ detected on chromosome 5 were consistent with those detected by Yoshioka et al. (2014) from the same resistant line, PI 197088. These two QTL may represent unique regions for DM resistance in PI 190788. However, multiple QTL are concentrated in another region on chromosome 5 (Szczechura et al. 2015; Win et al. 2016; Yoshioka et al. 2014; Zhang et al. 2013). These QTL regions are close to each other or overlapping (Fig. 2), which may be a major region for DM resistance in cucumber. We did not find DM resistance QTL in this region and the reason is not known.

Call (2012) suggested that, in PI 190788, DM resistance is controlled by at least three genes. Caldwell et al. (2014) and VandenLangenberg (2015) detected three QTL for DM resistance derived from PI 197088. Based on transcriptome analysis of DM-resistant and -susceptible materials, Burkhardt and Day (2016) provided evidence for the polygene hypothesis of DM resistance in PI 197088. In the present study, we detected a large number of QTL. For convenience, QTL with an overlapping area were considered to be the same. Among the five QTL (Table 2) detected in the present study, $d m 1.1$ was combined with two or three QTL regions, while $d m 5.2$ could be divided into two QTL. Thus, we believe that DM resistance in cucumber PI 197088 complies with the polygenic model and is controlled by more than three genes. However, this does not necessarily mean that the above resistance genes derived from PI 197088 are completely identical in all reports. More detailed in-depth studies are needed to clearly understand the genetic resistance of DM in PI 197088.

As a new source of DM resistance, PI 197088 is valuable for cucumber breeding. Thus far, no commercial varieties carrying DM resistance from PI 197088 are available in China. The QTL and molecular markers detected herein would be useful in marker-assisted selection in cucumber breeding for DM resistance. Nevertheless, more studies are needed to examine performance of PI 197088 for DM resistance under different environmental conditions (including temperature, humidity, and light), genetic populations, and plant growth stages, to explore QTL with stable and lasting resistance and further develop cucumber varieties with high DM resistance.

\section{Acknowledgments}

We thank Y. Weng (Horticulture Department, University of Wisconsin at Madison, WI) for providing information on SSR markers used in the present study.

\section{Literature Cited}

Angelov, D., Georgiev, P., Krasteva, L., Katzir, N., and Paris, H. S. 2000. Two races of Pseudoperonospora cubensis on cucumbers in Bulgaria. Acta Hortic. 510:81-84.
Arends, D., Prins, P., Jansen, R. C., and Broman, K. W. 2010. R/qtl: Highthroughput multiple QTL mapping. Bioinformatics 26:2990-2992.

Barnes, W., and Epps, W. 1954. An unreported type of resistance to cucumber downy mildew. Plant Dis. Rep. 38:620.

Broman, K. W., and Sen, S. 2009. A Guide to QTL Mapping with R/qtl. Springer, New York.

Broman, K. W., Wu, H., Sen, S., and Churchill, G. A. 2003. R/qtl: QTL mapping in experimental crosses. Bioinformatics 19:889-890.

Burkhardt, A., and Day, B. 2016. Transcriptome and small RNAome dynamics during a resistant and susceptible interaction between cucumber and downy mildew. Plant Genome 9.

Caldwell, D., Chan, E., De Vries, J., Joobeur, T., King, J., Reina, A., and Shetty, N. 2014. Methods and compositions for identifying downy mildew resistant cucumber plants. Google Patent US20110126309A1.

Call, A. D. 2012. Inheritance of resistance to downy mildew in cucumber (Cucumis sativus L.) PI 197088 and effect of interaction of host plant resistance, fungicides, and environment on severity of downy mildew on cucumber. North Carolina State University, Raleigh.

Call, A. D., Criswell, A. D., Wehner, T. C., Ando, K., and Grumet, R. 2012a. Resistance of cucumber cultivars to a new strain of cucurbit downy mildew. HortScience 47:171-178.

Call, A. D., Criswell, A. D., Wehner, T. C., Klosinska, U., and Kozik, E. U. 2012b. Screening cucumber for resistance to downy mildew caused by Pseudoperonospora cubensis (Berk. and Curt.) Rostov. Crop Sci. 52:577-592.

Cavagnaro, P. F., Senalik, D. A., Yang, L., Simon, P. W., Harkins, T. T., Kodira, C. D., Huang, S., and Weng, Y. 2010. Genome-wide characterization of simple sequence repeats in cucumber (Cucumis sativus L.). BMC Genomics 11:569.

Cohen, Y., Rubin, A. E., Galperin, M., Ploch, S., Runge, F., and Thines, M. 2014 Seed transmission of Pseudoperonospora cubensis. PLoS One 9:e109766.

Cohen, Y., Van den Langenberg, K. M., Wehner, T. C., Ojiambo, P. S., Hausbeck, M., Quesada-Ocampo, L. M., Lebeda, A., Sierotzki, H., and Gisi, U. 2015. Resurgence of Pseudoperonospora cubensis: The causal agent of cucurbit downy mildew. Phytopathology 105:998-1012.

Colucci, S. J., and Holmes, G. J. 2010. Downy mildew of cucurbits. Online publication. Plant Health Instruct. doi:10.1094/PHI-I-2010-0825-01

Fukino, N., Yoshioka, Y., Sugiyama, M., Sakata, Y., and Matsumoto, S. 2013. Identification and validation of powdery mildew (Podosphaera xanthii)resistant loci in recombinant inbred lines of cucumber (Cucumis sativus $\mathrm{L}$.) Mol. Breed. 32:267-277.

He, X. M., Li, Y. H., Pandey, S., Yandell, B. S., Pathak, M., and Weng, Y. Q. 2013. QTL mapping of powdery mildew resistance in WI 2757 cucumber (Cucumis sativus L.). Theor. Appl. Genet. 126:2149-2161.

Hoagland, D. R., and Arnon, D. I. 1950. The water-culture method for growing plants without soil. Calif. Agric. Exp. Stn. Circ. 347:357-359.

Holmes, G. J., Ojiambo, P. S., Hausbeck, M. K., Quesada-Ocampo, L., and Keinath, A. P. 2015. Resurgence of cucurbit downy mildew in the United States: A watershed event for research and extension. Plant Dis. 99:428-441.

Jenkins, J. M. 1942. Downy mildew resistance in cucumbers. J. Hered. 33:35-38.

Kosambi, D. D. 1943. The estimation of map distances from recombination values. Ann. Eugen. 12:172-175.

Lebeda, A., and Cohen, Y. 2011. Cucurbit downy mildew (Pseudoperonospora cubensis)-biology, ecology, epidemiology, host-pathogen interaction and control. Eur. J. Plant Pathol. 129:157-192.

Lebeda, A., Gadasová, V., Nishimura, S., Ezura, H., Matsuda, T., and Tazuke, A. 2002. Pathogenic variation of Pseudoperonospora cubensis in the Czech Republic and some other European countries. Acta Hortic. 588:137-141.

Li, Y., Wen, C., and Weng, Y. 2013. Fine mapping of the pleiotropic locus B for black spine and orange mature fruit color in cucumber identifies a $50 \mathrm{~kb}$ region containing a R2R3-MYB transcription factor. Theor. Appl. Genet. 126: 2187-2196.

Naegele, R. P., Quesada-Ocampo, L. M., Kurjan, J. D., Saude, C., and Hausbeck, M. K. 2016. Regional and temporal population structure of Pseudoperonospora cubensis in Michigan and Ontario. Phytopathology 106:372-379.

Ojiambo, P. S., Gent, D. H., Quesada-Ocampo, L. M., Hausbeck, M. K., and Holmes, G. J. 2015. Epidemiology and population biology of Pseudoperonospora cubensis: A model system for management of downy mildews. Annu. Rev. Phytopathol. 53:223-246.

Pais, M., Win, J., Yoshida, K., Etherington, G. J., Cano, L. M., Raffaele, S., Banfield M. J., Jones, A., Kamoun, S., and Saunders, D. G. 2013. From pathogen genomes to host plant processes: The power of plant parasitic oomycetes. Genome Biol. $14: 211$

Palti, J., and Cohen, Y. 1980. Downy mildew of Cucurbits (Pseudoperonospora Cubensis): The fungus and its hosts, distribution, epidemiology and control. Phytoparasitica 8:109-147.

Pang, X., Zhou, X., Wan, H., and Chen, J. 2013. QTL mapping of downy mildew resistance in an introgression line derived from interspecific hybridization between cucumber and Cucumis hystrix. J. Phytopathol. 161:536-543.

Ren, Y., Zhang, Z., Liu, J., Staub, J. E., Han, Y., Cheng, Z., Li, X., Lu, J., Miao, H., Kang, H., Xie, B., Gu, X., Wang, X., Du, Y., Jin, W., and Huang, S. 2009. An integrated genetic and cytogenetic map of the cucumber genome. PLoS One 4:e5795.

Sakata, Y., Kubo, N., Morishita, M., Kitadani, E., Sugiyama, M., and Hirai, M. 2006. QTL analysis of powdery mildew resistance in cucumber (Cucumis sativus L.). Theor. Appl. Genet. 112:243-250. 
Savory, E. A., Granke, L. L., Quesada-Ocampo, L. M., Varbanova, M., Hausbeck, M. K., and Day, B. 2011. The cucurbit downy mildew pathogen Pseudoperonospora cubensis. Mol. Plant Pathol. 12:217-226.

Shetty, N. V., Wehner, T. C., Thomas, C. E., Doruchowski, R. W., and Shetty, K. V. 2002. Evidence for downy mildew races in cucumber tested in Asia, Europe, and North America. Sci. Hortic. (Amsterdam) 94:231-239.

Szczechura, W., Staniaszek, M., Klosinska, U., and Kozik, E. U. 2015. Molecular analysis of new sources of resistance to Pseudoperonospora cubensis (Berk. et Curt.) Rostovzev in cucumber. Russ. J. Genet. 51:974-979.

VandenLangenberg, K. M. 2015. Studies on Downy Mildew Resistance in Cucumber (Cucumis sativus L.). North Carolina State University, Raleigh.

Van Ooijen, J. W. 2006. JoinMap® 4.0: Software for the Calculation of Genetic Linkage Maps in Experimental Populations. Kyazma B.V., Wageningen, Netherlands.

Voorrips, R. E. 2002. MapChart: Software for the graphical presentation of linkage maps and QTLs. J. Hered. 93:77-78.

Wallace, E., Adams, M., and Quesada-Ocampo, L. M. 2015. First report of downy mildew on buffalo gourd (Cucurbita foetidissima) caused by Pseudoperonospora cubensis in north Carolina. Plant Dis. 99:1861.

Wang, S., Basten, C., and Zeng, Z. 2012. Windows QTL Cartographer 2.5. North Carolina State University Statistical Genetics \& Bioinformatics, Raleigh. https://brcwebportal.cos.ncsu.edu/qtlcart/WQTLCart.htm
Wang, Y., VandenLangenberg, K., Wehner, T. C., Kraan, P. A. G., Suelmann, J. Zheng, X., Owens, K., and Weng, Y. 2016. QTL mapping for downy mildew resistance in cucumber inbred line WI7120 (PI 330628). Theor. Appl. Genet. 129:1493-1505.

Wehner, T. C., and Shetty, N. V. 1997. Downy mildew resistance of the cucumber germplasm collection in North Carolina field tests. Crop Sci. 37:1331-1340.

Weng, Y., Colle, M., Wang, Y., Yang, L., Rubinstein, M., Sherman, A., Ophir, R. and Grumet, R. 2015. QTL mapping in multiple populations and development stages reveals dynamic quantitative trait loci for fruit size in cucumbers of different market classes. Theor. Appl. Genet. 128:1747-1763.

Win, K. T., Vegas, J., Zhang, C., Song, K., and Lee, S. 2016. QTL mapping for downy mildew resistance in cucumber via bulked segregant analysis using next-generation sequencing and conventional methods. Theor. Appl. Genet. 130:199-211.

Yang, L., Li, D., Li, Y., Gu, X., Huang, S., Garcia-Mas, J., and Weng, Y. 2013. A 1,681-locus consensus genetic map of cultivated cucumber including $67 \mathrm{NB}-$ LRR resistance gene homolog and ten gene loci. BMC Plant Biol. 13:53.

Yoshioka, Y., Sakata, Y., Sugiyama, M., and Fukino, N. 2014. Identification of quantitative trait loci for downy mildew resistance in cucumber (Cucumis sativus L.). Euphytica 198:265-276.

Zhang, S., Liu, M. M., Miao, H., Zhang, S. Q., Yang, Y., Xie, B., Wehner, T., and Gu, X. F. 2013. Chromosomal mapping and QTL analysis of resistance to downy mildew in Cucumis sativus. Plant Dis. 97:245-251. 